\title{
Green cooperative communication network using renewable energy sources
}

\author{
Sanjay kumar, jaya diptilal, S.V charhate \\ Department of electronics and telecommunicationS.G.S institute of technology and science indore,india
}

\begin{abstract}
Financial and environmental considerations have motivated a trend in wireless communication network design and operation to minimize the amount of energy consumption . such a trend is reffered to as green radio communication. in this article, network cooperation is investigated as a means of energy saving. Network with overlapped coverage can cooperate to reduce their energy consumption by alternately switching on and off their resources according to the traffic load condition. We present an optimal resource on-off switching framework that adapts to the fluctuation in trafficload and maximize the amount of energy saving under service quality constrants, in a cooperative networking environment. For the system model inter consideration, unlike the existing solution in literature, the proposed technique can achieve energy saving while avoiding an increase in the transmission power, numerical results demonstrate the validity of the proposed technique.
\end{abstract}

Keywords: EARTH, gion, energy efficiency,reen communications,femtocells, interference,radio resource management.

\section{Introduction}

Telecommunication has experienced a tremendous success causing proliferaoration and demand for ubiquitous heterogeneous broadband mobile wirelass communications. Up to now, innovation aimed at improving wireless work coverage and capacity while meeting the QoS requested by users admitted to the system. Nowdays, the number of mobile subscribers equals more than half the global population . forecast on telecommunication market assumes an increase in subscribers, per subscriber's data rate and the roll out of out of additional base station for next generation mobile networks. An undesired consequence is the groath of wireless network's energy consumption that will cause an increase of the global carbon dioxide $(\mathrm{CO} 2)$ emission, and impose more challenging more operational cost for operators. Communication energy efficiency(EE) presents indeed an alarming bottleneck in the telecommunication growth paradigm. Motivated by this scenario, we outline the main investigation axes that may significantly improve the EE of broadband cellular networks, thereby reducing the cost and environmental impact of mobile broadband services. Today's mobile networks have a strong potential of energy savings. The design of mobile networks have been focused on reducing the energy consumption. This has led to a situation where terminal energy consumption is only a fraction of the energy consumption of the mobile network. For example. NTT DOCOMO has calculated that, for their 52 million subscribers in 2006, the energy consumption of their network per mobile user per day was 120 times greater than the daily energy consumption of NTT DOCOMO'S network over the year from 2002 to 2006.cosequently, the efficient optimization of energy consumption of radio base station should have a large impact on the overall $\mathrm{EE}$ of the network as a matter of fact, recent increasing maturity of mobile technology in combination with the growing amount of equipment deployed each year have woken up the need to innovate in the field of energy efficient communications.the main federative projects that face the challenge of enhancing the EE in mobile wirelessbroadband networks are the core 5 Green Radio programme of mobile virtual centre of excellence(MVCE), Green Touch and EARTH

Energy efficient enhancement in wireless communication can be achieved only if improvement are experienced in the whole communication chain for different operational load scenarios. Several investigations are carried out in this research area, raging from energy efficient cooling of base stations to investigate energy efficient deploympent stegies, and frequenc and frequency planning. The EARTH project setsa very innovative challenge: deployment of an holistic methodology to characterize the energy consumption of cellular networks including suitable matrics . today, there is no exists no widely agreed methodology to evaluate the energy efficiciency of cellular networks. Similarly mference eaningful energy efficient matrics are currently lacking. Both, in conjuction with reference scenarios, will enable a successful evaluation of the energy efficiency of networks. Such agreed methodology will be enabler of a fair comparison of different concepts and technologies.

The object of this paper is threefold. First, we present in detail the holistic system view proposed in the EARTH project, which may allow us to ensure that any proposed radio EE improvement does not degrade EE or of the whole communication system. We focus on how energy consumption of mobile broadband network may be significantly reduced in representative traffic scenarios. We consider LTE like systems which face a very 
challenging multi user communicatioin : many users in the same geographical area require on high on- demand data rates in finite bandwidth with a variety of heterogeneous services such as voice (VoIP), video,gaming, web browsing and others. We will present a holistic optimization methodology that includes socio-economic impact of broadband communication, definitioin of reference scenarios, and targets and evaluation metrics.

Second, we present and benchmark the EE effectiveness of a novel energy efficient ratio resource management(RRM) algorithm for OFDMA based systems. Since wireless is a shared medium, the system EE is affected not only by the single user efficiency, but also by the combination of time and frequency allocation according to the momentary instance of the of the frequency selective channel between the downlink base station and the receiving user equipments. Hence in order to achieve EE, a system approach is required. in our vision the multi -user scheduler should indeed allocate time and frequency resources to minimize the transmission energy cost while meeting QoS requirements of all active users admitted by a base station. The momentary system load plays an important role in the overall optimization design. Currently deployed base station are commonly designed so that they can accommodate the traffic demand at peak times. Nevertheless, cell traffic load notably varies during the day. Several researches pointed out that to save energy, base station should perform a dynamic load and energy state arrangement, thus maintaining minimum energy consumption . in this paper we do not propose novel dynamic sleep moode of base station components.we focus on resource allocation of active phases of base station s during downlink transmission. We propose a simple algorithm to trade off momentary spectral efficiency for downlink transmission power while meeting the QoS constraints of active users admitted in the cell. to this end, we propose a scheduling algorithm which splits the resource allocation process into four steps. In the first step we identify which entities(packets) are rushing and which are not rushing. Then in step two we deal with urgencies: we assign resource only to entities that have an high probability of missing their QoS requirements regardless to their momentary link quality and their potential to save energy. Than if any resource to user (non-rushing) with highest momentary link quality, regardless to their QoS constraints . in the fourth step we perform energy efficient link adaption to save downlink energy. we tradeoff throughput (lowering the transmission spectral efficiency and allocating a larger number of chunks to UEs) for downlink power by limiting the power budget on each chunk. In this way we attempt to minimize downlink transmission power over a time window, which provides a significant additional flexibility to the scheduling algorithm in an extreme case of latency to tolerance or low load scenarios, the scheduler could simply wait for the user to get close to the base station before transmitting or allocate all frequency resource to a single packet transmission with significant lowered power . we call the proposed algorithm a Green Adaptive Scheduler(GAS)

Third we propose an RRM algorithm to improve the EE of a heterogeneous cellular network where macrocells and femtocells[8]share the same spectrum in a given geographical area. In such heterogeneous deployment scenario, in terms of both spectral efficiency and energy efficiency.

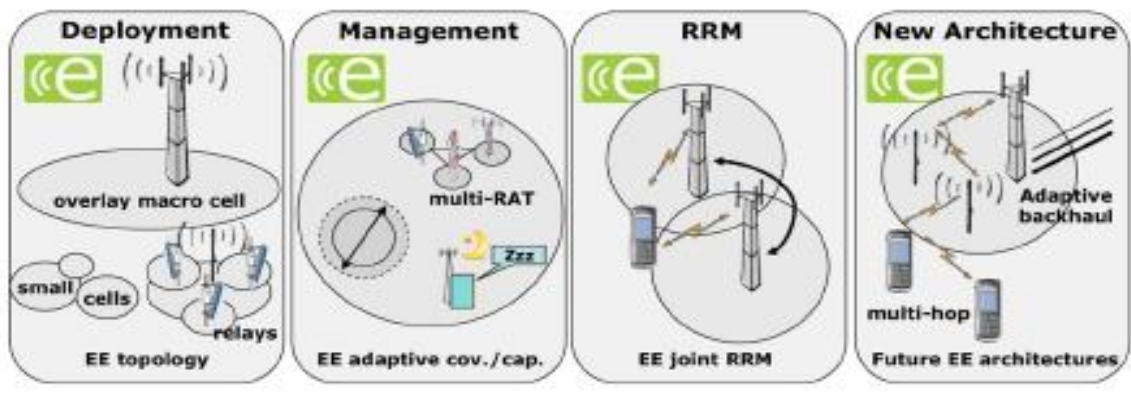

Figure 1 Green networks innovation axes

\section{Holistic EE Optimization Methodology for a Broadband Cellular Network}

A unified approach to improve the $\mathrm{EE}$ of the whole communication systemis still missing. Neverthe less, the potential offered by joint optimizations isin our vision the most promising one. Joint optimizations are represented bycross layer optimizations that take component and node architectures as wellas radio interface tech nologies and network architecture into account. Wepresent here the holistic optimization methodology propos ed in the EARTH

project that is composed of three major investigation axes:

. EE Optimization Methodology Framework

- EE Design of Green Networks

- EE Design of Green Radios 
These three investigation axes will be the background knowledge for the design of new green network architecture.

that should be investigated at network level.

Figure 1 summarizes the concepts

\section{Definition of an EE Optimization Methodology Framework}

Today running broadband cellular networks have been designed to maximizecoverage areas and spectr al efficiency of communication systems. In suchnetworks EE optimization has not yet played a prime rol e. Hence, metricslike spectral efficiency, capacity or throughput have been used to characterize usefulness an d differences of investigated solutions. Nevertheless, thesemetrics are not sufficient to judge the EE of a comm unication system. A fun

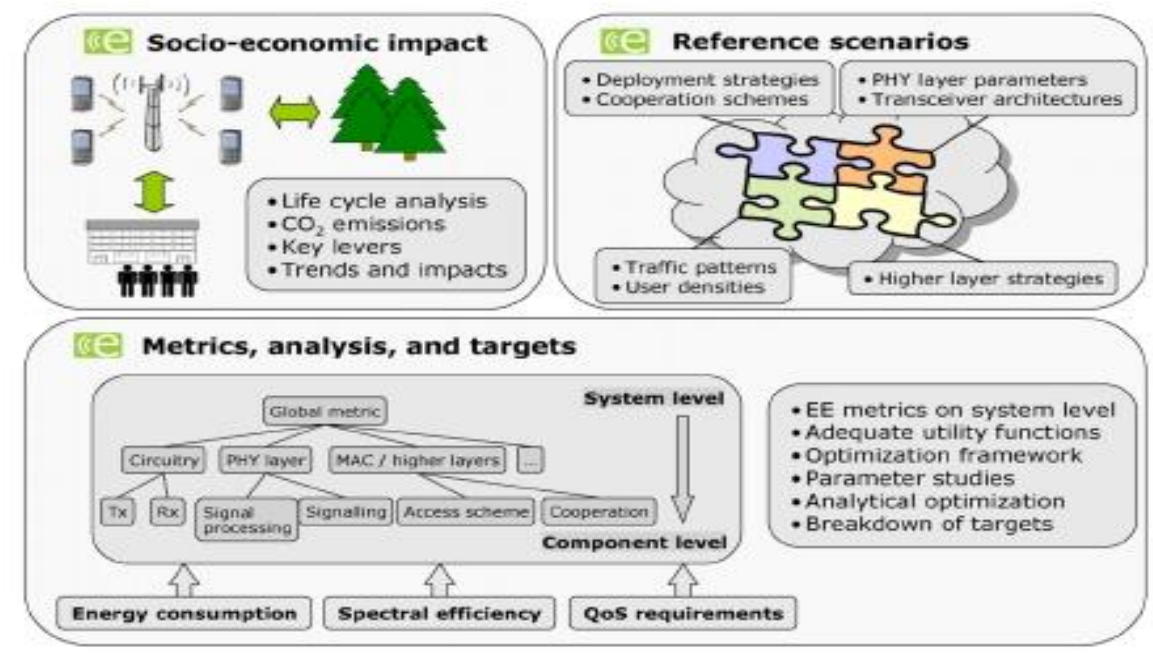

Figure 2 Framework to enhance the EE of broadband cellular networks

damental analysis of key radio technologies in terms of their EE is lacking.This is partly due to the fact that $t$ here are neither suitable methodologies nor metrics established to enable a fair and objective comparison of these techniques. Thus, an important prerequisite for efforts to enhance the EE ofcellular networks is to provide a framework to evaluate these technologies. Figure 2 we summarize the proposed workin $\mathrm{g}$ framework

First, the socio-

economic impact of broadband communication systempermits to define and forecast targets of energy efficient optimization. Then,the definition of reference scenarios characterizes the specific frameworkfor whic $\mathrm{h}$ some specific EE enhancements are envisioned. Eventually, to evaluate the effectiveness of the EE optimization, metrics are defined. Today there is not common view on such EE mettargets and evaluation rics in the wireless community and, a wide range of metrics are used byvendors operators and academi a. Since 2009, standardization of energy efficiency metrics has started. One of the major bodies of standardi zation thatis working on the topic is the ETSI Technical Committee on Environmental engineering

. Furthermore, criteria related to the quality-of service are neglected.Nevertheless, to achieve the goal of a holistic network design, EE metrics should also take the following into account:

- The input power required to generate a specific output power at theantennas. Energy consumed by digital signal processing equipment (interference cancelation techniques, complex decoding, etc.). With this type of met-rics, the tradeoff between EE improvement of specific signal processing algorithms and their consumed energy can be understood

- Specific metrics on EE of RRM schemes and their related control signalling cost. These types of metrics are worth when the EE analysis of the system considers not only the single-user optimization problem but also the multi-user case. With such a type of metrics we can understand the tradeoff between EE improvement due to multiuser RRMalgorithms (such as frequency and packet s cheduling, power allocationand power control, etc. ) and additional control signalling cost.

- The energy to deliver data to the base station (backhaul power consump-tion). 


\section{Design of Green Networks}

Until now, several Radio Access Technologies (RATs) have been deployed

in a parallel way, accommodating different service requirements over the same area and providing multiRAT handovers. Nevertheless, such solutionshave standalone network planning and resource management metho dologies.In our method, the common network design goal has been to optimize thes ystem's capacity and coverage while meeting QoS requirements of differ-ent types of applications in very different communication scenarios, ranging from large macro to femto cells for different environmental characteristics. Solutions have been optimized $f$ or the high load system operating point.

Nevertheless, systems are most of the time offloaded. Very recently efforts are originated for joint management of the multi-RAT technologies focus ing on common capacity and coverage optimization. These previous efforts did not include EE considerations optimal deployment or performance from an energy consumption point of view. Moreover, cellular networks exhibit slowly changing daily load patterns as well as highly dynamic traffic fluctuations. However, currently networksare configured rather statically. Since most of the energy wastage occurs during low load situations, these load variations can be effectively exploited to reduce network energy consumption. Therefore, dynamic load variations should be addressed by energy efficient RRM strategies. Innovative design ofgreen networks would requir to innovate in the following three topics

- Network Deployment.

- Network Management.

Radio Resource Management.

\subsection{Network Deployment}

While a lot of work has been performed on developing throughput, capacity or coverage enhancing conc epts for cellular networks, little insights have been gained which techniques can be applied to improve the energy efficiency resulting in less optimal strategies for overall network energy consumption. Moreover, recently the heterogeneous radio access networks(HetNets) concept is under investi gation by both academics and industry.The HetNet deployment proposes a flexible and open architecture for a 1 arge variety of wireless access technologies, applications and services with dif-

ferent QoS demands as well as different protocol stacks. Wireless networks differ from each other by air interface technology, cell-

size, services, price,access, coverage and ownership. The complementary characteristics offered by the different RATs make possible to exploit the deployment diversity gain leading to higher overall performance than the aggregated performances ofthe standalone networks. Actually, the biggest advantages of a heterogeneousnetwork are the significant gains in network capacity and coverage via aggr essive spatial spectrum reuse of available bands. This gain can be exploitedto drastically reduce the overall energy consumption of the network. To thisend, it is first fundamental to determine the theoretical and practical limitations on energy efficiency of HetNets deployment scenarios. The goal is to

understand how to reduce system energy consumption based on deploymentstrategies using different cell sizes, ne twork topologies, coordination betweenradio access technologies and investigates network deployments inclu dingmacro base stations, femto cells, relays and repeaters that share the samespectrum in the same geograp hical area. Then, the second challenge will beto define how to reduce overall system

energy consumption by combiningand coordinating different sites and radio technologies. This is the object o $\mathrm{f}$ EE RRM techniques. Eventually, there is the challenge of designing meth odologies for energy efficient radio network deployments including femtocells, relays, repeaters, distribute $\mathrm{d}$ antennas, etc. Indeed, an energy efficient network deployment can be design by defining the optimal mix of cell size,share of the common spectrum and, the set of adaptive resource allocation methods that may drive to large EE gains

\subsection{Network Management}

In our vision, innovative network management concepts will lead to large network's energy consumption savings. The challenge is to understand how to tune the radio network nodes in order to achieve gains from coordination between different functions and nodes in the radio network, including thebackbone network. The studied mechanisms should enable self EE optimization of the network, requiring only minimal human intervention. The concept is that the system autonomously and dynamically changes the cellular layout of the mobile network according to, e.g., the daily traffic profile 
To this end, the design of novel EE network management functions requires to investigate theoretical limits

of energy efficient network management algorithms. Then, it is required to understand how overall system

energy consumption can be reduced through proper configuration and tuningof radio network nodes, including interaction and cooperation between net-

work elements (including radio, transport and backbone network elements).

Furthermore, network management methods for EE multi-RAT coordination should be investigated.

\subsection{Energy Efficient RRM}

RRM algorithms have been designed to maximize the system capacity while

overcoming the mismatch between requested QoS and limited network resources under full system load. However, system load in mobile systems is dynamic in nature and, traditional RRM schemes are not necessarily efficient

at different operating conditions. Novel RRM schemes developed, in addition to load variation, should take into account the characteristics of RF front-end

such as Power Amplifiers (PA) as well as uplink and downlink power andbandwidth constraints. This is further enhanced by the development of RRM

cell cooperation for power control and scheduling schemes considering multiof admitted heterogeneous active users

\section{Design of Green Radios}

Additionally, novel technologies and components, as well as energy efficient radio interface techniques must be holistically investigated. Innovative design of green radios would require innovation of the following three topics

- Reduction of dissipated power of base station hardware components.

- Design of link interfaces and application of innovative radio transmis-sion techniques.

- Energy efficient RRM.

Figure 3 summarizes the concepts that we propose to investigate at node and single cell levels.

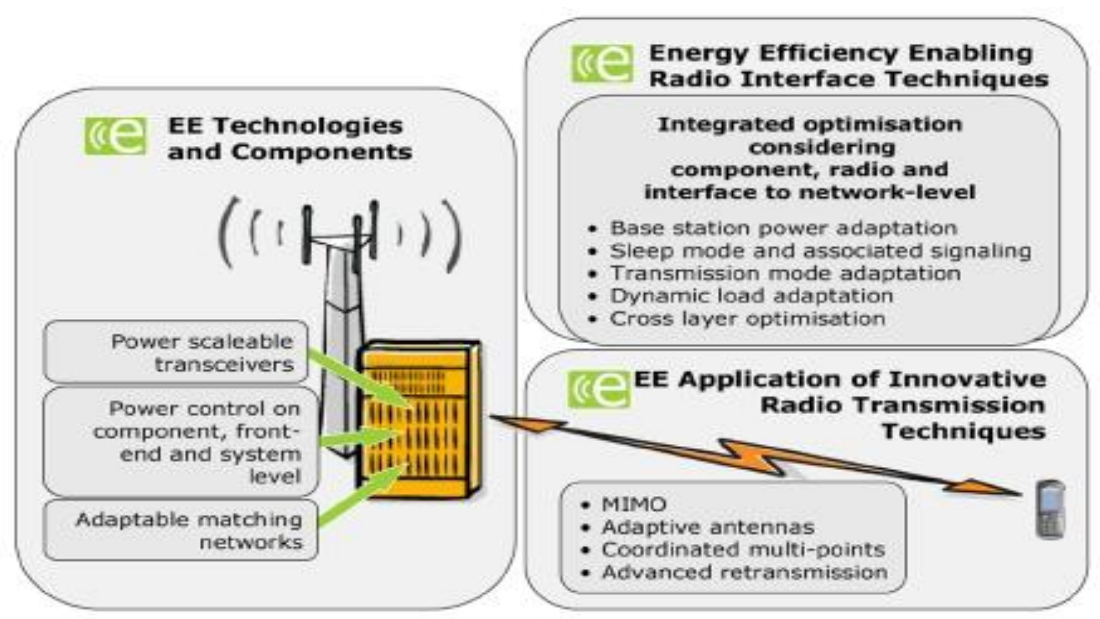

Figure 3 Green radios innovation axes.

\subsection{Reduction of Dissipated Power of Base Station Hardware Components}

In a green network, depending on the coverage range and momentary utilization ratio of base stations, different peak output power levels will be required. State of the art Power Amplifier (PA) do not full fill these requirements. Currently available solutions are optimized regarding the power efficiency in maximum load scenarios.

However, the maximum load scenario is a rather rare case in typical network deployments of operators That means that most of the time, the network infrastructure is operated with sub-optimal EE thus leading to a significant waste of energy resources. Therefore, power efficiency of base stations components has potential. Radical new approaches should be envisaged to realize funda- 
mental efficiency improvement. To this end, new configurations of PA should be investigated. Actually, PA should be

able to tune the output power for different operating conditions in real time. For instance, power variations at the output of the PA cause impedance variations resulting in antenna mismatch and thereby in power wastag e of the overall system.

In our vision, joint optimization of PA and antenna networks should be introduced to achieve

reconfigurable/tuneable interstate matching networks. Moreover, transceivers should support the scaling of the consumed energy in order to enable the

adaptation of energy consumption to actual performance requirements. Such

energy scalability should be present in all components (analogue RF

frontend, baseband) and in all devices. Furthermore, transceivers should enable dynamic power management in order $t$ o keep active only their necessary modules during sleep modes. Indeed, at component level, a key investigation axe is the design of traffic load adaptive base station components, with harmonized solutions for EE control on system level and energy efficient base station components unlocking solutions for EE operation of cellular networks.

\subsection{Design of Link Interfaces and Application of Innovative Radio Transmission Techniques}

solutions on isolated aspects and mainly non-cellular systems . A similar embracing analysis of the EE of radio link

technologies for cellular is lacking, despite cellular systems represent the major energy consumers due their universal proliferation. In particular, the aspects of the radio link operation in low load scenarios have not been addressed so far. In order to progress the state of the art on energy efficient radio link technologies, several State of the art radio link designs (such as beam forming, MIMO, adaptive antennas arrays, coordinated multi-point transmission and, advanced retrans mission schemes) have been designed to achieve high peak data rates, good coverage and low latencies. As consequence, mobile terminals require to operate periodic control messages and reference signals from base stations. This limits the possibilities for base station sleep intervals in low load scenarios. Consequently, a signi ficant amount of energy today is wasted by not having appropriate base station sleep mechanisms in place. Some researchers have started proposing investigation axes should be explored. First, innovation should achieve joint optimization of energy efficient radio link operation strategies for all load regimes. Second, short range coverage such as in femto cells deployment should be extensively investigated.

\section{Deployment of Femtocells and System Model}

In our analisys, we assume that femtocells are deployed according to the3GPP grid urban deployment mod el (see Figure 4). This model represents a single floor building with $10 \mathrm{~m} \times 10 \mathrm{~m}$ apartments placed next to each other in a $5 \times 5$ grid. The block of apartments belongs to the same regionof a macrocell.Moreover, we assu me that six additional MBSs surround thecentral macrocell generating additive interference for both macro and fe mtousers. Each HeNB can simultaneously serve up to four users. In order toconsider a realistic case in which some apartments do not have femtocells, we use a system parameter $\rho d$ called a deployment ratio t hat indicates the percentage of apartments with a femtocell. Furthermore, the 3GPP model

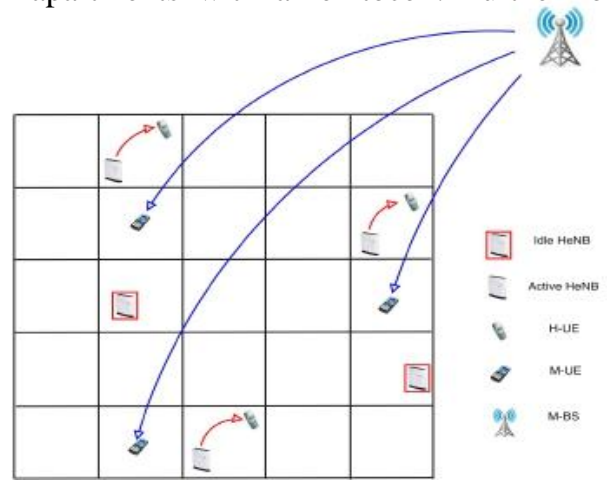

Figure 4 3GPP femtocell grid urban deployment model 
includes $\rho a$, another parameter called an activation ratio defined as the percentage of active femtocells. If a femtocell is active, it will transmit with a

certain power over data channels. Otherwise, it will transmit over the control

channel.

We use two different models to capture the signal propagation effect based

on the 3GPP specifications

1. Transmissions from femto and macro users to M-BSs:

$P L(d B)=15.3+37.6 \log 10 d+$ Low,

where $d$ is the distance between the transmitter and the receiver (inmeters) and Low is the

penetration losses of an outside wall equal to $20 \mathrm{~dB}$.

2. Transmissions from femto and macro users to HeNBs:

$P L(d B)=38.46+20 \log 10 d+0.7 d 2 D$, indoor

$+18.3 n((n+2) /(n+1)-0.46)+q \cdot$ Liw,

where $d$ is the distance between the transmitter and the receiver (in

meters), $d 2 D$,indoor is the two dimensional separation between the trans

Table 2 Main system model parameters.

Parameter

Carrier frequency

Carrier bandwidth

Total number of Resource Blocks

Inter-site distance

Access Technique

M-BS Tx power

M-BS antenna gain after cable loss

HeNB maximum Tx power

HeNB antenna gain after cable loss

Shadowing distribution

Shadowing standard deviation in the interfering links

Shadowing standard deviation in the femto/macro user useful link $4 / 8 \mathrm{~dB}$

Autocorrelation distance of shadowing

Fast fading distribution

Thermal noise density

$\mathrm{Hz}$
Value

$2.0 \mathrm{GHz}$

$10.0 \mathrm{MHz}$

50

$500 \mathrm{~m}$

OFDMA

$16 \mathrm{dBW}$

$13 \mathrm{~dB}$

$-17 \mathrm{dBW}$

$0 \mathrm{~dB}$

Log-normal

$8 \mathrm{~dB}$

$50 \mathrm{~m}$

Rayleigh

$N 0=-174 \mathrm{dBm} /$

mitter and the receiver (in meters), $n$ is the number of penetrated floors, $q$ is the number of walls that separate the us er apartments and the trans-mitting HeNB apartment, Liw is the penetration loss of walls within the

grid of apartments equal to $5 \mathrm{~dB}$. The third term in the above expression

represents the penetration loss due to walls inside an apartment. This attenuation is modeled as a loglinear value equal to $0.7 \mathrm{~dB} / \mathrm{m}$. The

fourth term represents the penetration loss within different floors. In the

single floor building scenario considered, we have $d=d 2 D$, indoor and $n=0$.

Table 2 shows key model parameters including shadowing, fast fading, the macrocell antenna gain, and the transmission power

\section{Simulation Results}

In this section, we assess the effectiveness of the proposed RRMGhost by

comparing its performance with a reference algorithm (RRMclassic). The maindifferences between these schemes are:

1. In RRMclassic, there is no coordination within the femtocell network.Hence, HeNBs are not aware o $\mathrm{f}$ the presence and allocation strategy of neighbour HeNBs

2. RRMclassic aims to maximize the spectral efficiency of femtocells while

minimizing the probability that users that

to same RBs. Thus, the RRMclassic attempts to limit the number of RBs

belong to different cells access

allotted to each H-UE

3. RRMclassic algorithm does not implement MCS and Power scaling (Steps 6 and 8 in RRMGhost algorithm) 


\section{Conclusion}

In this article, network cooperation as means of energy saving in green radio communication is investigated. For the system model with overlapped coverage from different network. The proposed technique can achieve energy saving without increasing the transmission power. It relies on the cooperation among defferent networks to save energy on two scales. On a large scale, network with overlapped coverage alternatively switch on and off their BSs according to the long term fluctuation in the traffic load. On a small scale, each active BS switches on and off its channels according to the short term fluctuation in the traffic load. Numerical results demonstrate a satisfactory service quality in terms of call blocking probability and a large percentage of energy saving for each network . in the proposed framework, the service quality constraints can be extended to include other metrics than call blocking probability, such as the minimum achieved throughtput for data application, delay jitter for video streaming application.

[1]. Vikram Chandrasekhar and Jeffrey G. Andrews, Alan Gatherer, Texas Instruments "Femtocell Networks: A Survey"communication magazine,IEEE,vol.46,pp.59-67,sept 2012

[2]. Shuguang Cui, Andrea J. Goldsmith, and Ahmad Bahai, "Energy-constrained Modulation Optimization”IEEE Trans.wireless commun.,vol.4,no.5,pp.2349-2360,sept.,2011

[3]. Qing Chen and Mustafa Cenk Gursoy "Energy Efficiency Analysis in Amplify-and-Forward and Decode-and-Forward Cooperative Networks"information sceinse and systems. $53^{\text {rd }}$ annual conference on ,vol.no.pp.811-816

[4]. Qing Chen and Mustafa Cenk Gurso"Energy-Efficient Modulation Design for Reliable Communication in Wireless Networks”WCNC,2011 IEEE.,vol.no.,pp.1-6,18-21 april 2011

[5]. Albrecht J. Fehske, Fred Richter, and Gerhard P. Fettweis "Energy Efficiency Improvements through Micro Sites in Cellular Mobile Radio Networks"PIMPRC 2011 IEEE $46^{\text {TH }}$ INTERNATIONAL SYNOPSYM on,vol.,no.pp1-6,april 2010

[6]. Muhammad Ismail and Weihua Zhuang "Network Cooperation for Energy Saving in Green Radio Communications"wireless communication,IEEE vol no.48.,no.11,pp.no.66-72,nov.2011

[7]. Aria nosratinia, dallas, todd E. Hunter "Green technology in cooperative communication network using coded cooperative method" IEEE communication magazine vol.no.4,october 2013

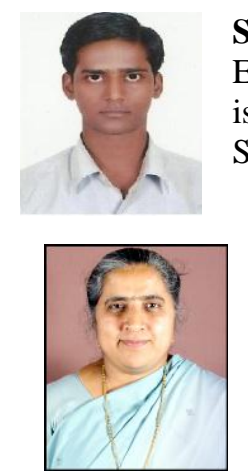

Sanjay kumar was born on $2^{\text {nd }}$ may 1989 in India. He received B.E degree in Electronics Engineering from Madhav institute of technology and science(MITS) Gwalior,India in 2011. He is currently studying M.E Degree in Electronics and Telecommunication S.G.S.I.T.S indore, India his research interest in wireless communication

Engineering from

Mrs. S.V Charhate was born on 14th may 1958 in India. She received B. E degree in Electronics and Telecommunication Engineering from college of engineering pune and M.E degree in computer science engineering from Shri G. S. institute of technology and science, indore . she has teaching experience of 26 years and currently Professor in the Department of Electronics and Telecommunicatio and Dean of Admn. In Shri G.S institute of technology and science indore, india . she has published papers in Nationa and International journals. Conferences and workshops organizsd by IEEE ,IETE and ISTE. Her research interest area in wireless communication.

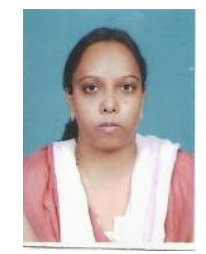

Mrs. Jaya diptilal was born on $1^{\text {st }}$ march 1977 in India. She received B.E degree(2002) in Electronics and Telecommunication from Jabalpur engineering college ,Jabalpur. And M.E degree(2005) from Jabalpur engineering college, Jabalpur. she has teaching experience of 7 years . she has published many papers in National and International Conferences, journals . her research interest area in cooperative communication , and green communication in cooperative radio network. 